children with a history of absence seizures (Clinical EEG April 1992; 23:62-64).

\title{
PERCEPTUAL MOTOR AND SENSORY INTEGRATIVE THERAPY FOR LEARNING DISABILITIES
}

The effects of sensory integrative therapy (SI), perceptual motor training (PM) and no treatment (NT) were compared in 103 children with learning disabilities at the Department of Paediatrics, Division of Neurology and Department of Rehabilitation Medicine, Hospital for Sick Children, University of Toronto, Canada. After a total of 72 1-hour sessions for 3 hours per week the PM treated subjects showed significant gains over the other 2 groups, primarily in gross motor performance, but without any accompanying carry over into functional activities such as copying ability, printing readiness, attention or organizational skills. SI treated subjects showed improvement in motor planning. Neither therapy resulted in improvement in cognitive, language or academic performance, attention or self-concept. (Humphries $\mathrm{T}$ et al. A comparison of the effectiveness of sensory integrative therapy and perceptual-motor training in treating children with learning disabilities. J Dev Behav Pediatr Feb 1992; 13:31-40.) (Reprints: Tom Humphries, Ph.D., Child Development Clinic, Hospital for Sick Children, Toronto, Ontario, Canada M5G 1X8.)

COMMENT. Although the claim that occupational therapy may directly improve higher level academic, language, and cognitive performance has not been supported by these studies, perceptual motor and sensory integrative therapy appears to have positive effects on motor planning and gross motor functioning. The possible value of these refinements of motor performance in effecting functional ability awaits further evaluation.

\section{SLEEP DISORDERS AND NEUROPSYCHOLOGICAL ABNORMALITIES}

The IQ and neurodevelopmental quotients of 17 of 32 children with congenital central hypoventilation syndrome were determined at the Departments of Pediatrics and Psychology, Rush-Presbyterian-St. Luke's Medical Center, Chicago, IL. Sleep hypoventilation was severe in all cases, with an alveolar carbon dioxide pressure of $62 \pm 2.5 \mathrm{~mm} \mathrm{Hg}$ and a hemoglobin saturation of $65 \%$. Seizures were associated in $72 \%$, pulmonary hypertension in $78 \%$ and mild cerebral atrophy in $40 \%$. All patients were hypotonic or had major motor delays. Autopsy performed in 6 cases showed diffuse central nervous system astrocytosis, gliosis, and atrophy but no brain-stem abnormality. The IQ and developmental quotients varied from greater than the 85 th percentile to less than the 5 th percentile, with verbal and performance discrepancies. The data support a diffuse central nervous system process but the possible effects of transient hypoxemia on neurodevelopmental test results could not be definitely determined (Weese-Mayer DE, Silvestri JM et al. I Pediatr March 1992; 120:381-7 and 388-93). (Reprints: Dr. Weese-Mayer, Department of Pediatrics, Rush-Presbyterian-St. Luke's Medical Center, 1653 West Congress Parkway, Chicago, IL 60612.) 
COMMENT. Congenital central hypoventilation syndrome, characterized by absent ventilatory and arousal sensitivity to hypercapnia and variable sensitivity to hypoxemia, has been reported with Hirschsprung disease, neural-crest tumors, and mild cerebral atrophy on CT or MRI. Many require ventilatory support particularly while asleep. Seizures and neurodevelopmental delays may bring these patients to the attention of the pediatric neurologist.

The effects of phenobarbital on sleep behavior in children with febrile seizures were evaluated by Hirtz DG, Farwell JR et al. at Bethesda, MD and Seattle, WA (Neurology April 1992; 42(Suppl $3): 367)$. Night awakenings were not more common in children on phenobarbital except for those who were poor sleepers initially. Lengthy night awakenings were not more frequent and total sleep time was not altered. At least one behavioral disturbance previously assigned to the use of phenobarbital in the prophylaxis of febrile seizures has been negated by this study.

Horne J from the Sleep Research Laboratory, Loughborough University, Leicestershire, U.K. discusses epilepsy in sleep in a paper on sleep and its disorders in children (J Child Psychol Psychiat March 1992; 33:473-487). In addition to febrile convulsions and benign rolandic childhood epilepsy, other paroxysmal disorders that occur during sleep include the complex partial seizures of frontal origin, usually misdiagnosed as nightmares (Stores G, 1991) and sometimes referred to as "nocturnal paroxysmal dystonia" (Lugaresi et al. 1986).

\section{PAROXYSMAL DISORDERS}

\section{PHENYTOIN AND NEUROPSYCHOLOGICAL PERFORMANCE}

A comprehensive neuropsychological assessment of young adults (mean age 32) before and after treatment with phenytoin (PHT) for a 5 year study period is reported from the Regional Epilepsy Center, University of Washington School of Medicine, Seattle, Washington. In 3 groups of 11 patients each, the following medications were not changed and the median number of seizures per subject was 2: (a) PHT monotherapy; (b) PHT and other drugs; (c) drug regimens excluding PHT. Of 20 neuropsychological variables, none showed losses and 3 showed statistically significant improvements (Trail Making Part B, WAIS Performance IQ, WAIS Full-Scale IQ). The mental abilities of the groups were similar to each other at both the beginning and the end of the study. With normal therapeutic serum levels and well controlled seizures there was no evidence for any cognitive losses in patients treated with phenytoin over extended periods. (Dodrill CB, Wilensky AJ.Neuropsychological abilities before and after 5 years of stable antiepileptic drug therapy. Epilepsia March/April 1992; 33:327-334.) (Reprints: Dr. C.B. Dodrill, Regional Epilepsy Center, Harborview Medical Center, 325 Ninth Avenue, Seattle, WA 98104.) 